\title{
FRÉQUENCE ET INTENSITÉ DU CHANCRE CYTOSPOREEN DANS LES PLANTATIONS D'ÉPINETTE DU QUÉBEC
}

\section{Par G. B. OUellette, J. M. CONWAY et G. BARD ${ }^{1}$}

En 1952, G. B. Ouellette obtient le degré de B.A. de l'Université SaintJoseph, Moncton, N.B., après quoi il termine deux années d'études à la Faculté d'Agriculture de la Pocatière, P.Q. En 1960, après des études post-universitaires au département de Pathologie végétale, il obtient le degré de Ph.D. de l'Université Cornell, Ithaca, N.Y. Il est à l'emploi du Laboratoire de Recherches forestières depuis 1958.

M.J. M. Conway obtint le degré de B.Sc. Agr. en sylviculture de la "National University of Ireland" en 1947. Venu au Canada en 1948, il remplit diverses fonctions avec la "Consolidated Paper Corporation Ltd." jusqu'en 1957, alors qu'on le nomme à sa situation actuelle de "Associate Research Forester".

Diplômé de l'Ecole des gardes-forestiers, Duchesnay, P.Q., M. Bard fut employé à la "Ste-Anne Power, Co.", de 1943 à 1956, Département de l'Exploitation forestière. Pendant la saison d'été, de 1952 à 1956, il remplit la fonction d'inspecteur garde-feu au service de la "Laurentian Forest Protective Association". En 1956, il passe au service du Laboratoire de Recherches forestières, ministère des Forêts du Canada, à titre de technicien en recherches forestieres.

\section{$A B S T R A C T$}

Cylospora canker was observed in all the plantations of white and Norway spruce examined in the Province of Quebec and in some natural stands of indigenous spruce species. Disease incidence was particularly high in the Grand'Mère and Saint-Lazare plantations where it has existed since at least 1943 and 1955, respectively. Favorable periods for infection occurred at fairly regular intervals and development of cankers was more pronounced some years than others. No clear-cut correlation was found between rainfall, temperature, and disease intensity. The disease is becoming more prevalent and poses a threat to existing spruce plantations in Québec.

\section{EXPLICATION DES FIGURES}

FIGURE 1. Précipitation estivale (de mai à seprembre inclusivement) et ríparticion du nombre d'arbres infectés et de nouvenux chancres selon les années.

FIGURE 2. Avance du champignon dans les couches externes de l'écorce. Les flèches montrent des bandes de tissus phellogìnes.

FIGURE 3. Envahissement progressif du tronc, surtout visible au point indiqué par la flèche, d'un chancre âgé de 13 ans.

FIGURE 4. Recouvrement d'un chancre sur une épincte blanehe à croissance remarquable et régulière.

FIGURE 5. Entrée d'une caric (probablement causéc par le Fomes pini) au niveau d'un chancre.

${ }^{1}$ Chargés de recherche. Ministère des Forêts du Canada, C.P. i5, Sillery 6, P.Q. 
SAINT-LAZARE
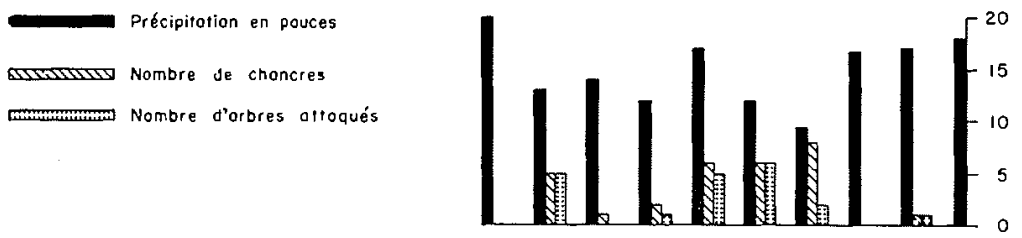
GRAN D'MĚrE
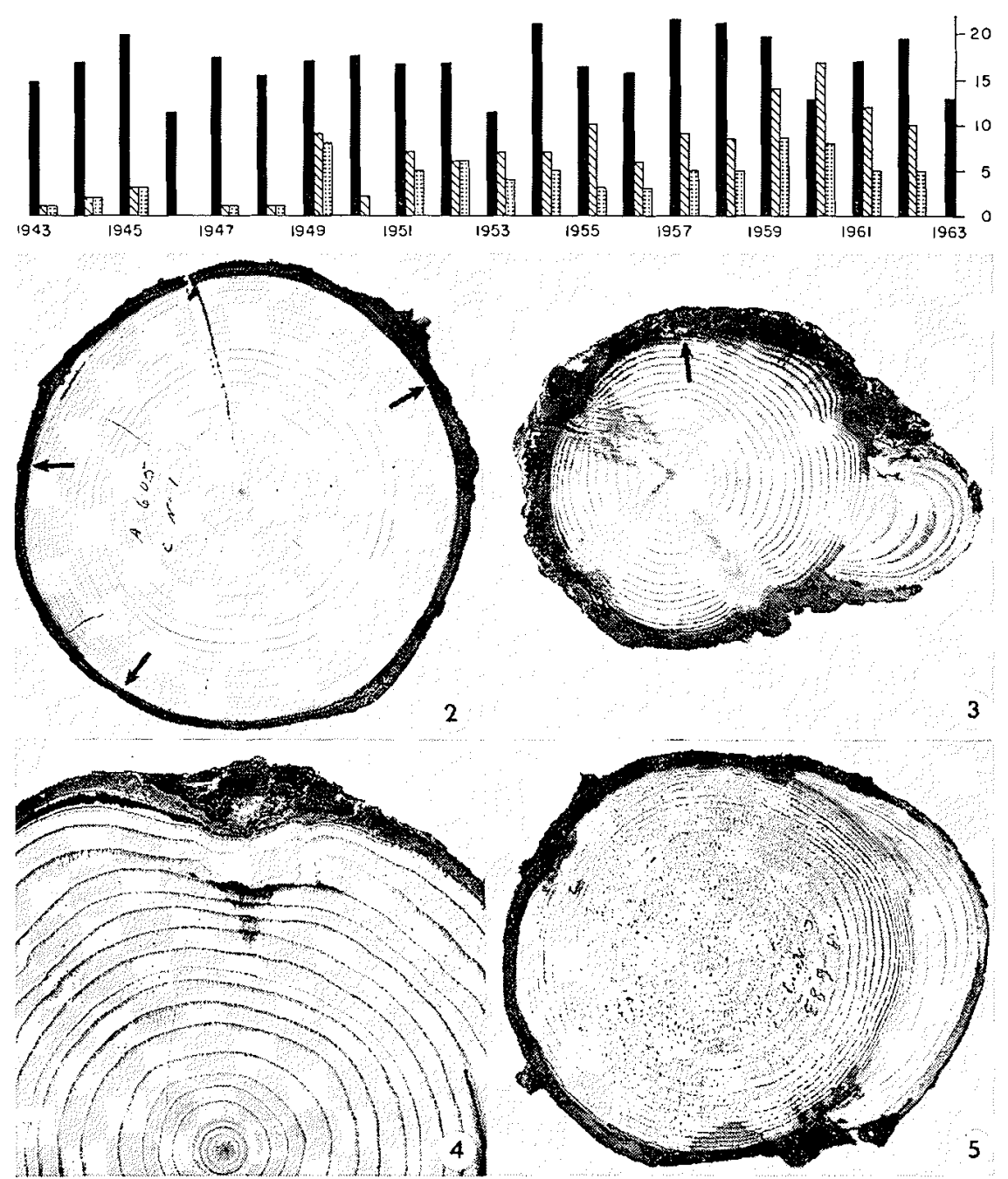
SOMMAIRE

Le chancre cytosporéen de l'épinette sévit souvent à l'état épidémique dans les plantations d'épinette blanche et d'épinette de Norvège visitées au Québec et s'y trouve aussi en quelques peuplements naturels. La maladie remonte à 1943 dans les plantations de Grand'Mère et à 1955, à Saint-Lazare, où l'on constate les plus forts pourcentages d'arbres attaqués. Nous avons noté des recrudescences du mal à intervalles assez réguliers au cours des années, alors que vraisemblablement certaines conditions ont favorisé tantôt l'avance, tantôt le retrait des chancres. A ucun rapport bien tranché ne semble exister entre ces fluctuations, les facteurs climatiques ou la croissance des arbres. Cette maladie prend de l'ampleur et semble pouvoir compromettre l'avenir des plantations au Québec.

\section{INTRODUCTION}

Depuis les quelques quarante années que l'on connaît l'existence en Amérique du Nord du chancre cytosporéen de l'épinette, causé par une espèce du genre Valsa, on le rencontrait surtout sur les épinettes ornementales (Gilgut et Boyd, 1933). Dernièrement, on a rapporté des cas graves de cette maladie dans des plantations d'épinette blanche, Picea glauca (Moench) Voss, et d'épinette de Norvège, Picea abies (L.) Karst, en Ontario et au Québec (Jorgensen et Cafley, 1961; Ouellette et Bard, 1962). Nous exposons ici les résultats de recherches sur la distribution, la fréquence et le développement de cette maladie dans le Québec.

\section{CONDITIONS dE RECHERCHE}

Environ 125 arbres atteints à différents degrés de gravité dans les plantations (Consolidated Paper Co. Ltd.) situées près de Grand'Mère (Laviolette), et une trentaine d'autres dans la plantation de Saint-Lazare (Vaudreuil), servirent à nos études. Lors de l'abattage, nous avons mesuré la hauteur, le diamètre et la longueur de la flèche terminale de ces arbres, prélevé des rondelles à la base du tronc, à la hauteur de poitrine ainsi qu'à la base et au sommet du cimier, et pris soin de mesurer l'étendue de tous les chancres du tronc, ou autres anomalies. Les valeurs de croissance annuelle furent obtenues en mesurant l'épaisseur des anneaux le long de quatre rayons tracés à angles droits sur les rondelles. Afin d'obtenir l'indice de l'avance ou du retrait des chancres, nous avons établi au point d'entrée de ceux-ci la dimension de chaque anneau de croissance envahi par le pathogène ou recouvert par les tissus cicatriciels (fig. 3 et 4 ).

Les données d'inventaire proviennent de l'examen de 2 ou 3 rangées d'arbres prises au hasard, sauf le cas de l'épinette blanche dans les plantations de Grand'Mère, où nous avons eu recours à des parcelles-échantillons circulaires d'un vingtième d'acre. Ces parcelles furent distribuées au hasard dans les stations de différentes qualités.

La présence du pathogène fut confirmée en l'isolant de tissus malades prélevés sur environ 10 p. 100 des arbres ou par la présence de ses fructifications sur un grand nombre d'autres.

Distribution et Fréquence de la Maladie

La maladie se rencontre dans toutes les plantations d'épinette blanche et d'épinette de Norvège visitées au cours de ces travaux. En outre, nous 
avons trouvé la maladie sur les trois essences indigènes d'épinette (Picea rubens Sarg., Picea mariana (Mills) B.S.P., et Picea glauca) en différents endroits de la province. Dans les plantations de 25 ans et plus, environ 5 p. 100 des arbres étaient affectés à Lachute (Argenteuil); plus de 35 p. 100 à Saint-Lazare; et de 4.9 à 32.7 p. 100 selon la qualité des stations dans les plantations de Grand'Mère (tableau 1). Le nombre d'arbres affectés atteignait 85 p. 100 par endroits. A proximité de la rivière Saint-Maurice, 7.9 et 19.8 p. 100 des arbres des deux meilleures classes de hauteur étaient attaqués comparativement à 3.8 et 3.3 pour les mêmes catégories d'arbres aux endroits plus éloignés de ce cours d'eau (moyenne d'un minimum de 6 parcelles dans chaque cas). Le pourcentage d'épinettes de Norvège atteintes ne dépassait pas 5 p. 100. Malgré cette proportion plutôt faible, l'attaque fréquente des plus beaux sujets eñ accroît l'importance.

Dans les autres plantations, dont plusieurs âgées de moins de 10 ans (La Patrie, Gould et Sawyerville (Compton), Shannon (Portneuf), Ayer's Cliff (Stanstead), Harrington (Argenteuil), et Lemieux (Lotbinière)), nous n'avons constaté que des cas isolés de la maladie.

Chronologie et Progres des Attaques

Dans les plantations de Grand'Mère, la maladie remonte au moins à 1943, et à 1955, à Saint-Lazare. Le nombre de chancres et d'arbres infectés a augmenté par bonds assez brusques aux deux endroits (fig. 1). Si l'on répartit par classes de hauteur les épinettes blanches attaquées à Grand Mère, on constate qu'un nombre progressivement plus grand d'arbres des classes supérieures contractent la maladie (tableau 2). Parfois, le champignon ne s'attaque qu'à l'écorce et il devient alors difficile de déterminer l'année de l'attaque. De cette façon, il

TABLEAU 1

FREQUENCE DU CHANCRE CYTOSPOREEN SELON LA QUALTTE DES STATYONS (Classes I a IV ${ }^{1}$ ) - EPINETTE BLANChe, GRAND'MERe, P.Q.

\begin{tabular}{|c|c|c|c|c|}
\hline $\begin{array}{c}\text { Diametre } \\
\text { (en pouces) }\end{array}$ & $\begin{array}{c}\text { Nombre } \\
\text { d'arbes is l'acre }\end{array}$ & $\begin{array}{c}\text { Nombre d'arbres } \\
\text { attaques }\end{array}$ & $\begin{array}{l}\text { Diametre moyen } \\
\text { des arbres }\end{array}$ & $\begin{array}{c}\text { Pourcentage } \\
\text { d'arbres attaques }\end{array}$ \\
\hline \multicolumn{5}{|c|}{ Classe I (18 parcelles) } \\
\hline De 1 à 3 & 94 & 0 & & \\
\hline De 4 à 7 & 714 & 41 & & \\
\hline De 1 à 12 & 925 & 45 & 5.8 & 4.9 \\
\hline \multicolumn{5}{|c|}{ Classe II (11 parcelles) } \\
\hline De 3 à 5 & 875 & 111 & & \\
\hline De 2 à 6 & 231 & 23 & & \\
\hline De 2 à 9 & 1167 & 138 & 4.3 & 11.8 \\
\hline \multicolumn{5}{|c|}{ Classe III ( 9 parcelles) } \\
\hline De 3 à 5 & 615 & 114 & & \\
\hline De 1 à 8 & 768 & 133 & 4.3 & 17.3 \\
\hline \multicolumn{5}{|c|}{ Classe IV (18 parcelles) } \\
\hline De 2 à 4 & 452 & 167 & & \\
\hline De 1 à 7 & 606 & 198 & 3.7 & 32.7 \\
\hline
\end{tabular}

${ }^{1}$ Basées sur des différences de hauteur et de densité des arbres. 
TABLEAU 2

Distribution des Arbres Infectes par Classes de Hauteur EPinette Blanche, Grand'Mere, P.Q.

\begin{tabular}{|c|c|c|c|c|c|c|c|c|}
\hline $\begin{array}{c}\text { Annees } \\
\text { d'infection }\end{array}$ & $\begin{array}{l}\text { de } 10 \\
\text { a } 15\end{array}$ & $\begin{array}{r}\text { de } 15 \\
\text { a } 20\end{array}$ & $\begin{array}{l}\text { de } 20 \\
\text { a } 25\end{array}$ & $\begin{array}{l}\text { Hantev } \\
\text { de } 25 \\
\text { a } 30\end{array}$ & $\begin{array}{c}\text { n pieds } \\
\text { do } 30 \\
\text { a 35 }\end{array}$ & $\begin{array}{l}\text { de } 35 \\
\text { a } 40\end{array}$ & $\begin{array}{l}\text { de } 40 \\
\text { a } 45\end{array}$ & $\begin{array}{l}\text { de } 45 \\
\text { a } 50\end{array}$ \\
\hline $1943-1946$ & & & & 1 & 1 & & & \\
\hline $1947-1950$ & 1 & 3 & 3 & 4 & & 1 & & \\
\hline $1951-1954$ & 1 & 5 & 1 & 2 & 2 & 7 & 2 & 1 \\
\hline $1955-1958$ & & 2 & 5 & 7 & 5 & 5 & 2 & 1 \\
\hline $1959-1962$ & 3 & 2 & 2 & 9 & 9 & 12 & 5 & 2 \\
\hline Total & 5 & 12 & 11 & 23 & 17 & 25 & 9 & 4 \\
\hline
\end{tabular}

peut s'implanter progressivement en plusieurs endroits, envahir subitement le cambium à tous ces points et tuer rapidement l'arbre (fig. 2). Hopkins (1963) a aussi noté la pénétration, à travers l'écorce intacte, du champignon (Atropellis piniphila (Weir) Lohman and Cash) responsable du chancre atropelléen du pin à aiguilles tordues. Cependant, Jorgensen et Cafley (1961) n'ont pas réussi à produire l'infection des épinettes par le Cytospora sans pratiquer de blessures.

A Saint-Lazare, les chancres ont généralement moins progressé la première année de l'infection que pendant les années suivantes. Dans les plantations d'épinette blanche de Grand'Mère, cependant, sauf quelques reprises d'activité des chancres de 5 à 9 ans, la situation inverse a existé notamment chez les arbres de hauteur intermédiaire (tableau 3). A ce dernier endroit, la cicatrisation a progressé à une allure de plus en plus vive selon l'ordre ascendant de hauteur des arbres, comme l'indiquent les données du retrait des chancres. Si l'on regroupe les sujets malades selon l'étage, on constate que les chancres des arbres intermédiaires ont progressé le plus rapidement et ceux des dominants, le moins rapidement. Cependant, il se peut que certains sujets intermédiaires et co-dominants appartenaient à une classe supérieure au moment de l'attaque de la maladie.

L'analyse des données selon les classes de diamètre (DHP) montre que les chancres progressent plus rapidement sur les arbres de 3 à 4 pouces que sur les autres (tableau 4).

La majorité des chancres ont cessé de s'accroître en largeur après un certain temps et seulement 16 des 110 chancres dans cet état (plantations de Grand' Mère), dont 9 sur les arbres intermédiaires et 6 sur les arbres co-dominants, ont repris leur activité. Cependant, l'apparition de nouveaux chancres et l'allongement des autres semblent neutraliser dans l'ensemble les effets de ce recouvrement (tableau 5). Les chancres se sont apparemment allongés à un rythme uniforme, sauf sur les arbres intermédiaires et opprimés où ils ont envahi le tronc sur une plus grande distance l'année de l'infection. Selon l'ordre décroissant des classes de hauteur des arbres, ils ont atteint en moyenne 9 à 28 pouces de longueur après une période moyenne de 2 à 2.5 ans de développement (calculée d'après l'avance radiale). Par ailleurs, la persistance 


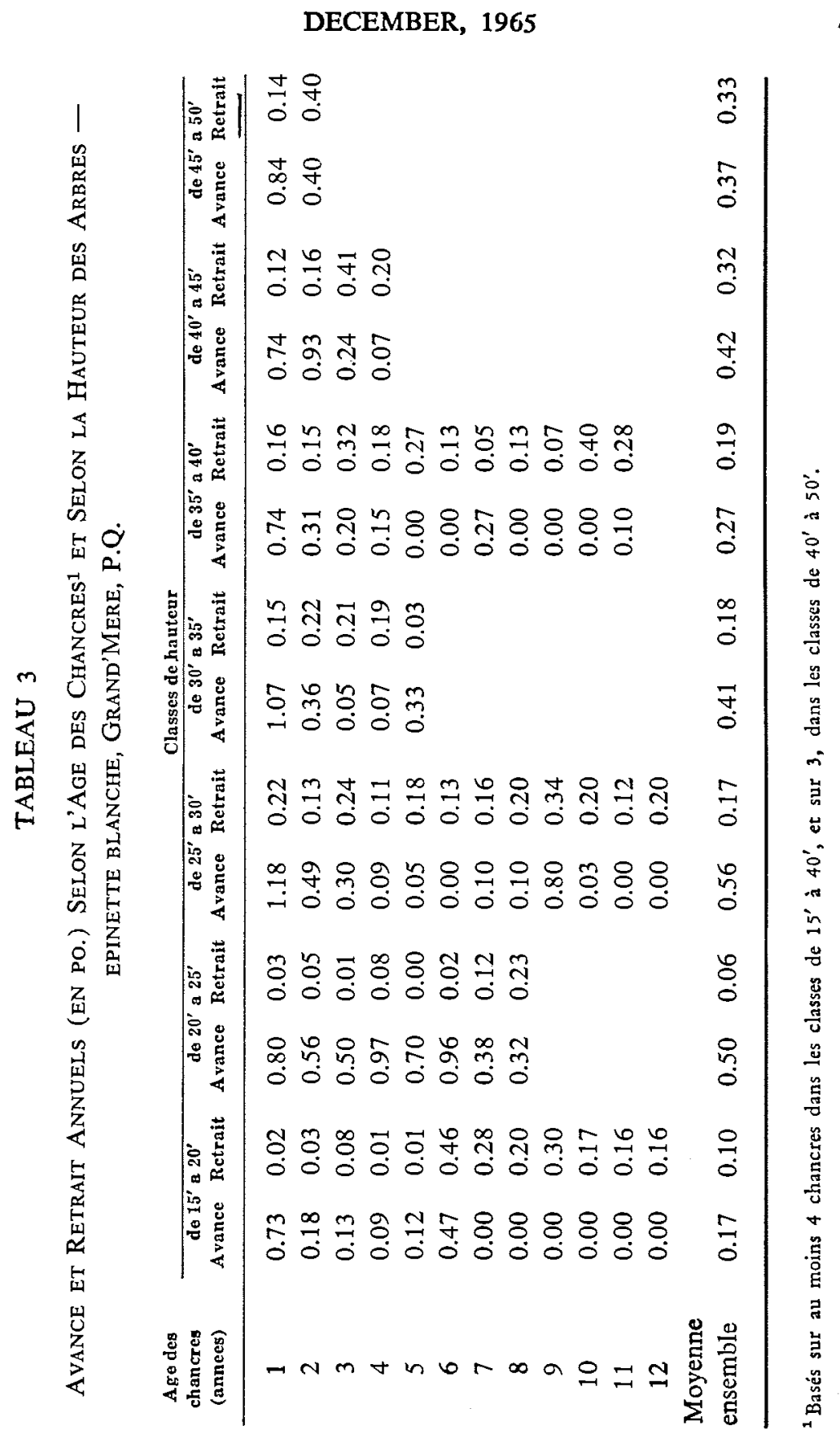


TABLEAU 4

Progres Radial et Cicatrisation des Chancres Selon les Classes de Diametre des Arbres, Grand'Mere, P.Q.

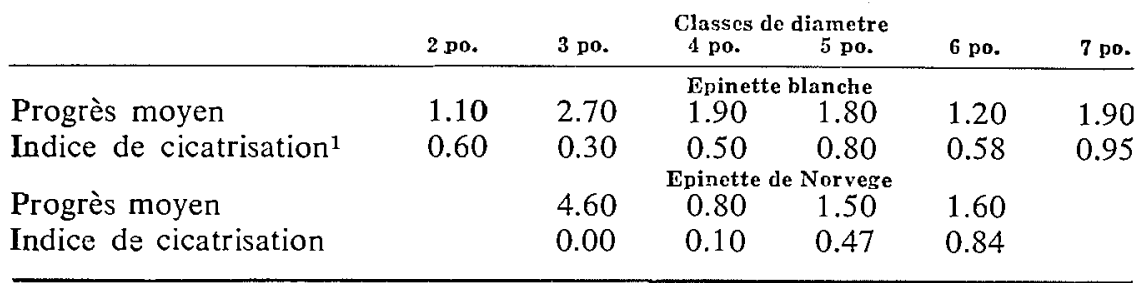

Obtenu en divisant la valeur de cicatrisation totale par la valeur de progrès total.

TABLEAU 5

Relation Entre Les Symptomes, L’Age, Le Nombre, et La Longueur DES CHANCRES

\begin{tabular}{ccccc} 
Groupo & \multicolumn{1}{c}{ Symptomes } & $\begin{array}{c}\text { Nombre moyen } \\
\text { d'annees depuis } \\
\text { la.premiere } \\
\text { infection }\end{array}$ & $\begin{array}{c}\text { Nombre moyen } \\
\text { de chancres } \\
\text { par arbre }\end{array}$ & $\begin{array}{c}\text { Longueur moyenne } \\
\text { des chancres en } \\
\text { pouces }\end{array}$ \\
\hline B & $\begin{array}{l}\text { Léger écoulement de } \\
\text { résine, feuillage } \\
\text { normal }\end{array}$ & 5.00 & 1.69 & 10.80 \\
C $\quad \begin{array}{l}\text { Ecoulement de résine } \\
\text { abondant, un peu de } \\
\text { feuillage jaune } \\
\text { Ecoulement de résine } \\
\text { abondant, feuillage } \\
\text { jaune ou considérable- } \\
\text { ment réduit }\end{array}$ & 7.60 & 2.09 & 12.28 \\
D & 8.00 & 2.50 & 21.20 \\
\hline
\end{tabular}

du champignon dans les couches externes đe l'écorce minimise les effets de la cicatrisation.

L'avance et le retrait des chancres ont été assez uniformes sur les arbres des différentes stations. Par exemple, dans la meilleure et la moins bonne station, l'avance annuelle moyenne s'établit dans l'ordre à 0.29 et 0.31 pouces, et le retrait, à 0.15 et 0.16 pouces.

La majorité des chancres ont progressé horizontalement plus vite certaines années que d'autres (tab'eau 6). Dans l'ensemble, pendant les années 1949 et 1952 à 1955 les conditions ont été particulièrement favorables à l'avance des chancres dans les plantations de Grand'Mère. Après 1955, on constate aussi une plus grande avance moyenne certaines années, mais celles-ci ne coineident pas toujours d'une classe de hauteur à l'autre. Ainsi paraissent les années 1957 et 1959 pour les arbres intermédiaires, 1959 à 1960 pour les co-dominants et 1957 à 1958 pour les dominants. A Saint-Lazare, l'avance annuelle des chancres (aussi plus prononcée certaines années) paraît le double 
TABLEAU 6

Avance et Retratt Moyens (en po.) des Chancres Selon Les Annees D'Attaque

\begin{tabular}{ccccc} 
Annees & \multicolumn{2}{c}{ Grand'Mere $^{\text {Grand }}$} & Setrait & \multicolumn{2}{c}{ Avance $^{\text {Saint-Lazare }}$} & Retralt \\
\hline 1963 & & & 0.19 & 0.13 \\
1962 & 0.31 & 0.19 & 0.70 & 0.00 \\
1961 & 0.26 & 0.18 & 0.40 & 0.23 \\
1960 & 0.20 & 0.20 & 0.60 & 0.15 \\
1959 & 0.30 & 0.16 & 0.52 & 0.23 \\
1958 & 0.25 & 0.17 & 0.61 & 0.13 \\
1957 & 0.29 & 0.20 & 0.90 & 0.34 \\
1956 & 0.27 & 0.15 & 0.87 & 0.31 \\
1955 & 0.44 & 0.11 & 0.48 & 0.02 \\
1954 & 0.51 & 0.11 & & \\
1953 & 0.39 & 0.06 & & \\
1952 & 0.46 & 0.07 & & \\
1951 & 0.32 & 0.15 & & \\
1950 & 0.29 & 0.05 & & \\
1949 & 0.75 & 0.01 & & \\
\hline
\end{tabular}

${ }^{1}$ Moyenne de 14 à 88 chancres.

Moycnne de 6 à 23 chancres.

ou le triple de celle des plantations de Grand'Mère. Ceci peut expliquer pourquoi les arbres à Saint-Lazare succombent en général plus vite à l'attaque. L'élagage pratiqué là en 1959 a peut-être favorisé la marche de la maladie.

\section{Rapport entre la Croissance et la Maladie}

D'après l'allure des courbes de croissance obtenues pour les arbres sains et attaqués dans les plantations de Grand'Mère, on peut confirmer la réduction générale de croissance assez subite et prolongée déjà rapportée par Paine (1960). De 1955 à 1962, la croissance a augmenté graduellement mais non sans certains fléchissements. On note que 57 p. 100 des chancres s'implantèrent tôt après la première chute de croissance, ou une autre par la suite, et 30 p. 100, plusieurs années plus tard, tandis que les autres attaques se sont produites sur des arbres à croissance uniforme (fig. 3 et 4 ) ou ont débuté après une reprise appréciable de croissance. Nous avons obtenu des données semblables pour l'épinette de Norvège, mais l'oscillation des courbes de croissance avec des pics plus élevés que les précédents souvent n'a pas permis de préciser le rapport entre la pénétration du pathogène et la croissance de l'arbre. A Saint-Lazare, par contre, la plupart des chancres sont apparus immédiatement après une chute de croissance. Antérieurement à l'attaque, la croissance des sujets attaqués aux deux endroits était aussi bonne sinon meilleure que celle des arbres demeurés indemnes, comme Jorgensen et Cafley (1961) l'ont observé aussi en Ontario. 


\section{Pertes}

Outre la carie assez commune des sujets affectés (fig. 5), la déformation des tiges (fig. 3 ) et éventuellement la mort des arbres, la maladie a provoqué dans les plantations de Grand'Mère une réduction déjà appréciable d'un pied de longueur totale de l'arbre et aurait abaissé en moyenne en 1962 de 3.5 pouces la longueur de la flèche terminale.

\section{INFLUENCE DU Climat}

La fiuctuation annuelle du nombre d'arbres infectés et du nombre de nouveaux chancres (fig. 1), et le progrès nettement plus grand de ceux-ci certaines années (tableau 6) indiquent l'influence de facteurs climatiques sur le développement de la maladie. L'analyse de le précipitation moyenne pour les mois de mai à septembre inclusivement montre une certaine analogie de rythme entre les fiuctuations de la précipitation et celles du progrès de la maladie. Sauf quand le nombre d'arbres infectés a augmenté subitement durant une année pluvieuse précédée d'années de faible précipitation, notamment en 1949, 1954 et 1957 , on ne saurait établir de rapport plus précis entre ces facteurs. Cependant, le nombre et la progression radiale des chancres a semblé s'accroître le plus durant les années de faible précipitation. La température bien au-dessus de la normale enregistrée de 1943 à 1949 et en 1955 près de Grand'Mère et en 1955, 1959 et 1960 à Saint-Lazare, a accompagné souvent des périodes de faible précipitation, par conséquent de sécheresse. Cependant, dans ce cas aussi, on ne peut rattacher à ces facteurs seuls le progrès de la maladie, parce que l'intensité de celle-ci semble décliner certaines de ces années ou augmenter de façon subite en d'autre temps. Il convient de signaler ici que l'existence du pathogène dans les branches et l'écorce externe pendant un certain temps, avant d'engendrer un chancre sur le tronc lui-même, peut compliquer singulièrement l'étude de ce problème.

\section{Discussion et CONCLUSIONS}

De cette étude, il ressort principalement que le chancre cytosporéen de l'épinette existe dans les plantations au Québec depuis au moins 1943. Du reste, des observations et des photographies contenues dans un rapport de Cosens, Haddow, Hasil et Phipps (1953) permettent de supposer la présence de cette maladie depuis déjà un certain temps dans les plantations de Grand'Mère. Nos données montrent aussi que la maladie se trouve dans toutes les plantations examinées, souvent sur des sols que plusieurs considèrent appauvris et peu appropriés à l'épinette, mais aussi sur des sols vraisemblablement beaucoup plus favorables. Dans ce dernier cas, cependant, le pourcentage d'arbres atteints de la maladie paraît beaucoup moins élevé, tandis que le recouvrement. s'avère aussi plus prononcé.

Selon Jorgensen et Cafley (1961), l'abaissement de l'humidité dans le sol et l'enracinement superficiel prédisposent les arbres à l'attaque du Cystospora sur l'épinette. Or, comme l'a démontré Paine (1960), une telle situation existait à Grand'Mère entre 1940 et 1953. Lavallée (1964) relie aussi à la sécheresse le développement du chancre cytosporéen sur le mélèze, causé par le Leucostoma kunzei (Fr.) Munk ex Kern. Dans le cas présent, on ne peut reconnaître de rapport bien défini entre la précipitation et le développement de la maladie, 
mais il semble exister un certain rapprochement des modes de fluctuation des deux. La recrudescence de la maladie lors d'années pluvieuses généralement précédées d'années de sécheresse peut s'expliquer par la plus grande quantité d'inoculum produite sous ces conditions, multipliant ainsi les chances d'infection d'arbres déjà affaiblis. On sait que l'effet de la sécheresse sur la croissance de l'arbre se fait sentir seulement l'année suivante (Gagnon, 1961). Par ailleurs, il faudrait ne pas méconnaître l'influence du climat hivernal sur le développement de la maladie, comme le suggèrent les observations de Robak (1964). La plus grande fréquence de la maladie et son développement plus rapide sur les arbres intermédiaires s'expliquent peut-être par l'influence de la densité des arbres dans cette classe (tableau 1). D'après Jorgensen et Cafley (1961), la trop grande densité du peuplement dans certains cas peut favoriser le développement de la maladie. La relation entre la croissance et la maladie, par contre, n'apparaît pas de façon certaine, surtout si l'on considère l'augmentation du nombre d'arbres infectés depuis 1955 en même temps qu'un regain de croissance de ceux-ci. En outre, la maladie semble s'attaquer de plus en plus aux sujets les plus vigoureux. Peut-être s'établit-il ici une sélection de races plus virulentes du pathogène.

Ces résultats montrent l'importance grandissante de cette maladie surtout dans les plantations, et indiquent la nécessité de définir davantage les conditions favorables à l'infection afin de trouver les moyens de répression appropriés.

\section{REMERCIEMENTS}

Nous désirons remercier M. Jean Smith, ministère des Terres et Forêts, province de Québec, d'avoir permis la coupe d'arbres dans la plantation de Saint-Lazare. Notre gratitude va aussi à M. Robert Gagnon, photographe, et à MM. René Pomerleau, D. E. Etheridge, Pierre Cochaux et Camilien Gagnon pour leurs conseils dans la préparation de ce manuscrit.

\section{RËFERENCES}

COSENS, G. A., W. R. HADDOW, R. C. HASIL et G. W. PHIPPS. 1953. Inspection of Laurentide Plantations. Mimeo.

GAGNON, DANIEL. 1961. Rainfall and the width of annual rings in planted white spruce. For. Chron. 37: 96-101.

GILGUT, C. J. et O. C. BOYD. 1933. Cytospora canker on Picea spp. (Abstr.) Phytopathology $23: 11$.

HOPKINS, J. C. 1963. Atropellis canker of lodgepole pine: etiology, symptoms, and canker development rates. Can. Jour. Botany 41: 1535-1545.

JORGENSEN, E. et J. D. CAFLEY. 1961. Branch and stem cankers of white and Norway spruce in Ontario. For. Chron. 37: 394-404. .

LAVALLÉE, A. 1964. A larch canker caused by Leucostoma kunzei (Fr.) Munk ex Kern. Can. Jour. Botany 42: 1495-1502.

OUELLETTE, G. B. et G. BARD. 1962. Observations on a canker and resinosis of white and Norway spruce in plantations. Bi-monthly Prog. Rept. For. Ent. \& Path. Br., Dept. For. Can. 18 (2): 2

PAINE, L. A. 1960. Studies in Forest Pathology. XXII. Nutrient deficiencies and climatic factors causing low volume production and active deterioration in white spruce. Can. Dept. Agr. For. Biol. Div. Public. 1067, 29 pp.

ROBAK, H. 1964. Some observations on larch canker and climate. FAO/Forest Pest-64. FAO/IUFRO Symposium on internationally dangerous forest diseases and insects. Oxford. July 20-30, $8 \mathrm{pp}$. 